- The face is the most recognisable feature of the body.

- Philosophers, scientists and sociologists have assigned many personality traits to facial features.

- Numerous ideas exist for linking facial landmarks to determine and influence the shape of the maxillary anterior teeth.

- Two key reference lines, interpupillary and incisal, are crucial for anterior aesthetics.

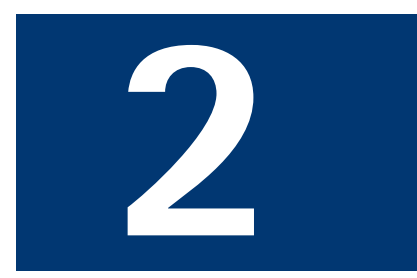

VERIFIABLE CPD PAPER

\title{
Anterior dental aesthetics: Facial perspective
}

\author{
I. Ahmad ${ }^{1}$
}

The purpose of this series is to convey the principles governing our aesthetic senses. Usually meaning visual perception, aesthetics is not merely limited to the ocular apparatus. The concept of aesthetics encompasses both the time-arts such as music, theatre, literature and film, as well as space-arts such as paintings, sculpture and architecture.

\section{ANTERIOR DENTAL AESTHETICS \\ 1. Historical perspective \\ 2. Facial perspective \\ 3. Dento-facial perspective \\ 4. Dental perspective \\ 5. Gingival perspective \\ 6. Psychological perspective*}

\footnotetext{
* Part 6 available in the BDJ Book
} of this series

\section{INTRODUCTION}

The face is the first view requiring assessment by a dental practitioner. This view not only reveals the physical landmarks of a person's identity, but also gives clues to their psychological make-up or persona. ${ }^{1}$ In addition, from an evolutionary standpoint, the face is the most recognisable feature of the body, an innate and learned response commencing in early childhood. ${ }^{2}$ Facial expressions mirror our emotional states and serve as crucial non-verbal communication tools. Facial muscle contractions convey feelings of fear, joy, happiness, anger, etc. without uttering a single word. Depending on the surrounding soft tissue envelope of the lips and cheeks, showing anterior teeth can signify pleasure (by a smile), or disdain (by a sneer). These examples illustrate the importance of teeth to the facial composition, serving the functions of mastication, communication and social interaction. Consequently, analysis of facial features influences dental restorations, particularly in the anterior region, by integrating with existing skeletal and soft tissue features to either enhance desirable qualities or distract attention from undesirable abnormalities.

${ }^{1}$ BDS, The Ridgeway Dental Surgery, 173 The Ridgeway, North Harrow, Middlesex HA2 7DF, United Kingdom.

Tel: +44 (0)20 88613535 , Fax: +44 (0)20 8861 6181, www.IrfanAhmadTRDS.co.uk Email:iahmadbds@aol.com

\section{Refereed Paper}

doi: $10.1038 /$ sj.bdj.4812534

(๑) British Dental Journal 2005; 199 :

15-21 professionals. The optical illusion of a white band of teeth bordered by red lips is constantly put forward in society, where a perfect smile is depicted as a monolithic band of ivory (Fig. 1). In reality, the upper anterior teeth are not a white monolithic band bordered by lips, but distinct entities with specific proportions and embrasures. These facts should be clearly conveyed to the patient at the onset of treatment to avoid later disagreements. The reason for the apparent illusion of the smile is due to:

- Angle and distance of view

- Lighting

- Profound colour contrast between teeth and the lips

- Poor image quality or

- Image manipulation.

There are several ways of creating facial assessment including physiognomic, morphopsychological and geometric. The first two

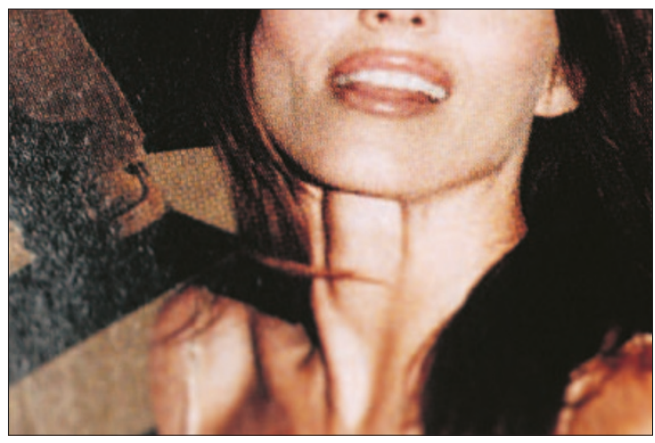

Fig. 1 Media representation of a smile as a monolithic band of ivory bordered by lips 


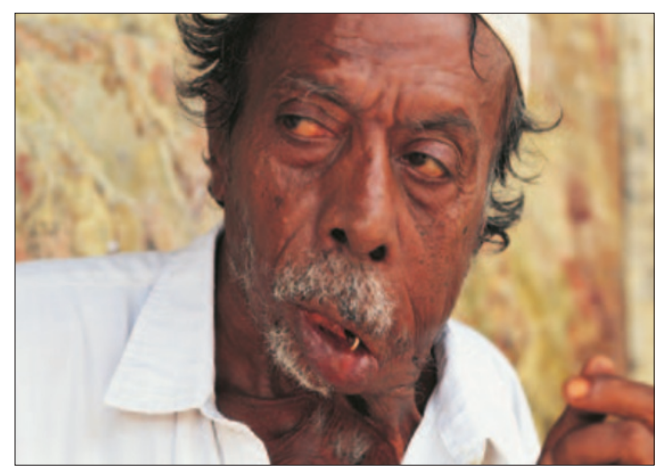

Fig. 2 In the third world, missing teeth are associated with poverty and misery

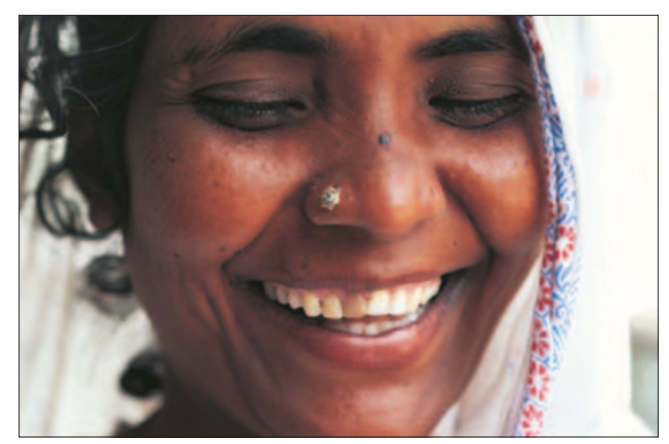

Fig. 3 In the developing world, discoloured teeth are synonymous with misfortune

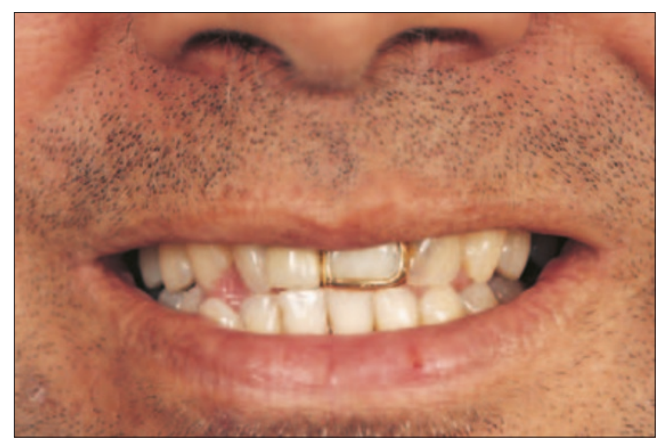

Fig. 4 In some African cultures, anterior gold dental prostheses signify prosperity

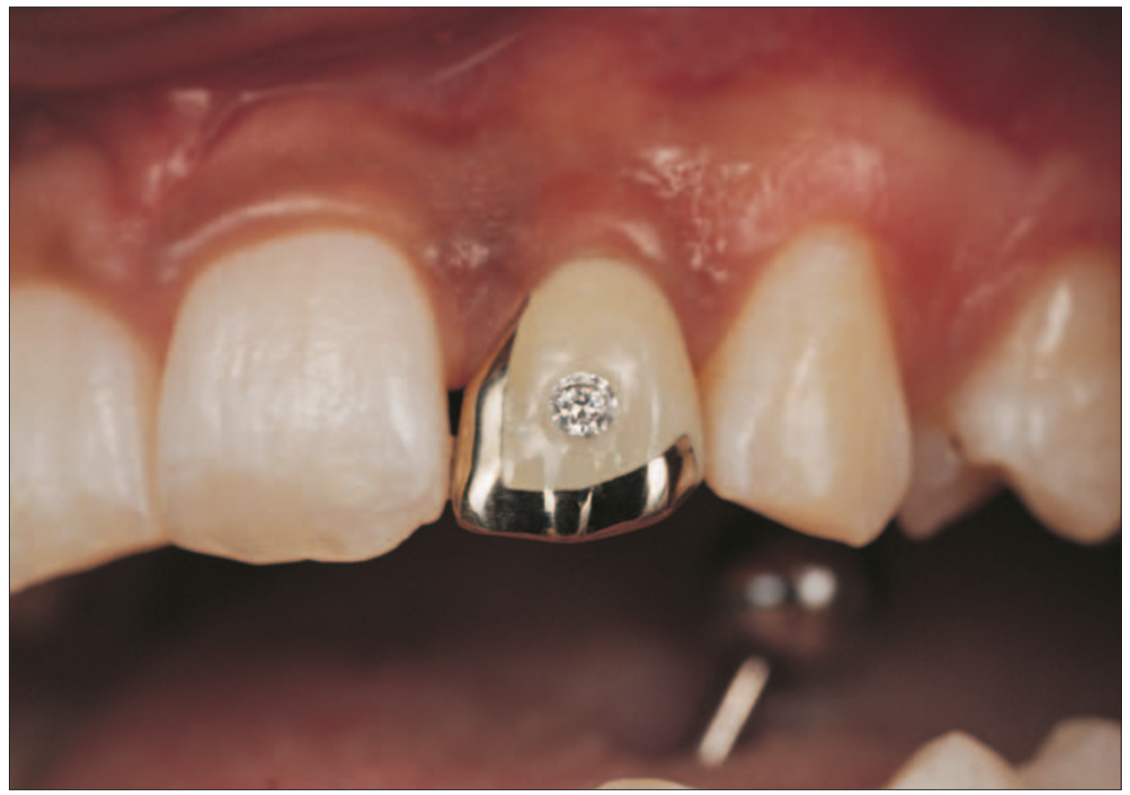

categories are subjective evaluations based on ideology, theology, sociology, culture and individuality, while conversely, geometric appraisal is an objective evaluation dictated by mathematics, devoid of idiosyncrasies inherent in the first two categories.

\section{PHYSIOGNOMY}

The facial perspective is the deciding factor for whether a treatment is a success or failure. The reason is that from this view, the patient, his/her family and friends make a physiognomic judgement regarding an individual's character. Physiognomy is the art of judging an individual's character or personality by the appearance of their face. This skill shares similar features with palmistry and astrology, dating back to antiquity, forming part of our collective consciousness. Although the relevance and meaning of facial features vary, physiognomic assessment is prevalent in Eastern and Western cultures. ${ }^{3}$ Whilst this may seem superficial, it is the commonest way of social assessment.

Objectively, the notion that facial features are responsible for a person's morality is at best spurious, and at worst stultifying. However, what is significant is that objectivity does not exclusively influence our daily lives. Rather, our behaviour is predominantly manipulated by emotional responses. ${ }^{4}$ This being the case, physiognomic judgement, which is a subjective assessment, profoundly affects social interaction.

It follows that if physiognomy plays a part in assessing people, the teeth, which are important facial landmarks, must also sway our judgement. This is termed dentofacial physiognomy. In the USA, for example, a bright white smile signifies affluence, youth, health, and promotes career progression. While in Europe, less emphasis is placed on a 'plastic' smile for a fulfilling life. These trends are simply indigenous aspects of two cultures; neither is right nor wrong, but merely different.

In Asian and Chinese societies, the number, size, colour and type of dental restorations are deciding factors of an individual's status and even destiny. ${ }^{5}$ In the developing world, missing incisors are synonymous with poverty and despair, while discoloured teeth portend disaster and misfortune (Figs 2 and 3). Another example is that Africans regard having anterior yellowgold prostheses as a sign of prosperity (Fig. 4). Finally, fashion trends and adulation inspire emulation, e.g. precious stones embedded into anterior teeth (Fig. 5).

The relevance of this discussion on dentofacial physiognomy is to raise cultural awareness of ethnic minorities among dental health providers. Offering ethnic minority patients dental restorations based on Western mores will alienate them from their culture and standing in community. And this will ultimately deter these patients from seeking further dental care for themselves and their families. 


\section{MORPHOPSYCHOLOGY}

The study of morphopsychology ${ }^{6}$ involves establishing a link between the morphology of the human body with psychological make-up. In dentistry, the face is the focus of attention, and the ensuing discussion on morphopsychology is therefore limited to facial features. The face is a reflection of our inner soul, influenced by both heredity and environmental factors. The former is beyond our control, while the latter is controllable by our will and certitude. Facial analysis is assessed by the following factors:

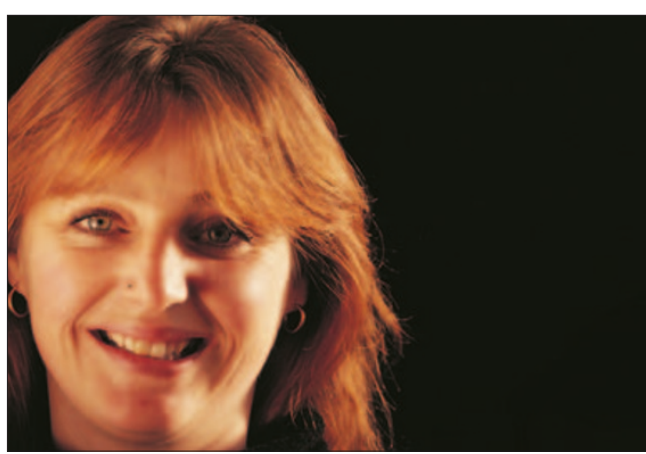

Fig. 6 Facial typology: lymphatic personality

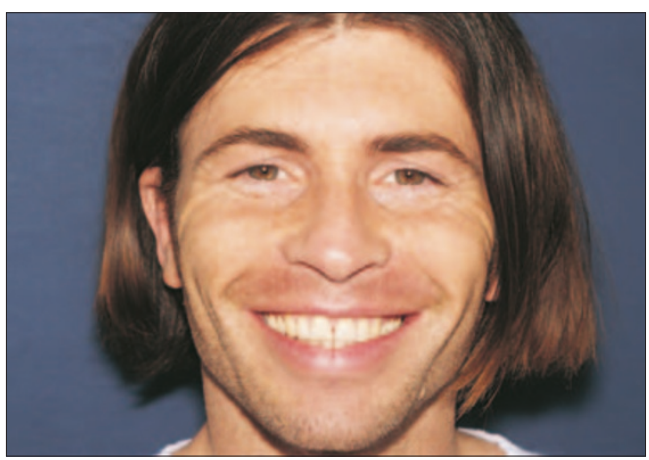

Fig. 7 Facial typology: sanguine personality

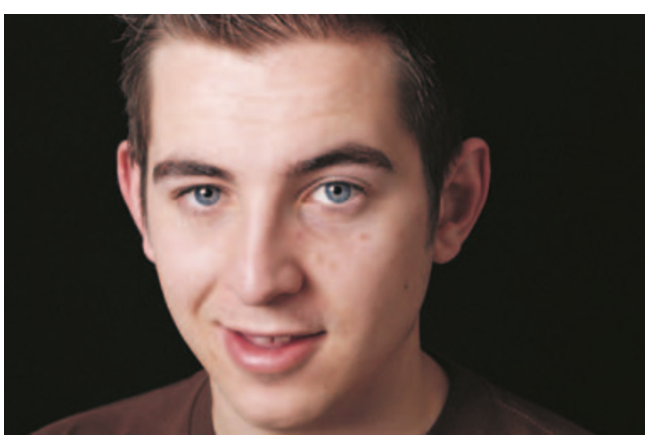

Fig. 8 Facial typology: nervous personality

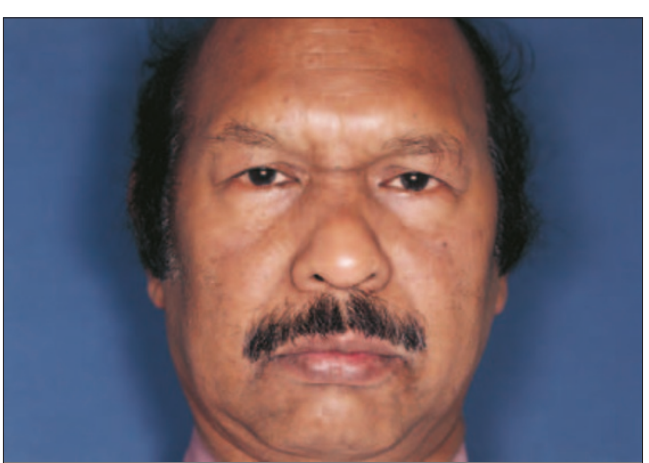

Fig. 9 Facial typology: bilious personality
- Facial typology

- Facial zones and segmental expansion

- Sensitive receptors

- Tegumental texture and relief

- Sexual type

- Hemifaces.

From a typological perspective, faces are assigned to one of four categories (Figs 6-9):

- Lymphatic (rounded full features with a timid personality)

- Sanguine (prominent thick well-defined features associated with intransigence and spontaneity)

- Nervous (large forehead, thin delicate features with an anxious disposition)

- Bilious (rectangular and muscular features coupled with a dominant persona).

The form of teeth should conform to these four types, so that for example, providing delicate or fragile looking teeth for a bilious face is clearly incongruous.

In both the frontal and sagittal views, the face is divided into three zones: upper, middle and lower. The upper segment is from the hairline to the glabella (root of the nose), the middle from the root of the nose to the subnasale, and the lower from the subnasale to the soft tissue menton (chin prominence) (Figs 10 and 11). This morphological differentiation signifies specific psychological traits: the upper third is associated with creativity, the middle with emotions and the lower with instinctive and sensual activities.

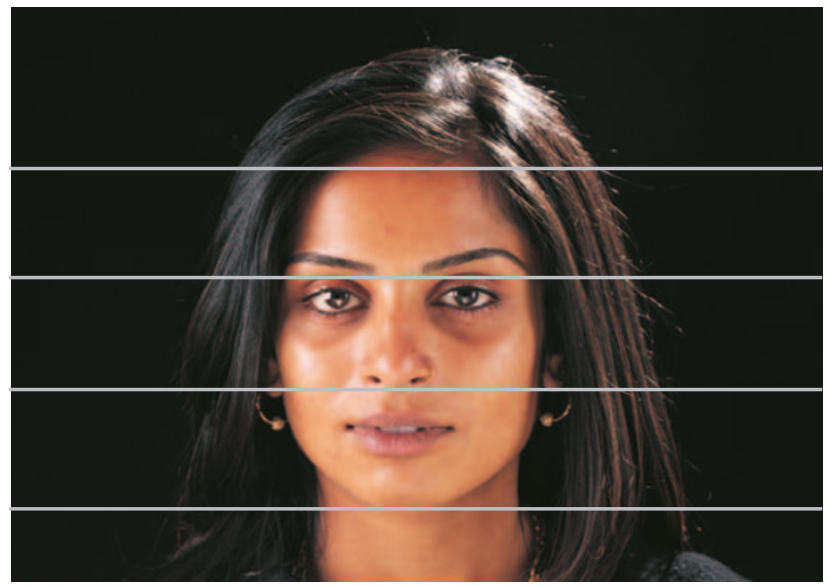

Fig. 10 Frontal view of the three facial divisions

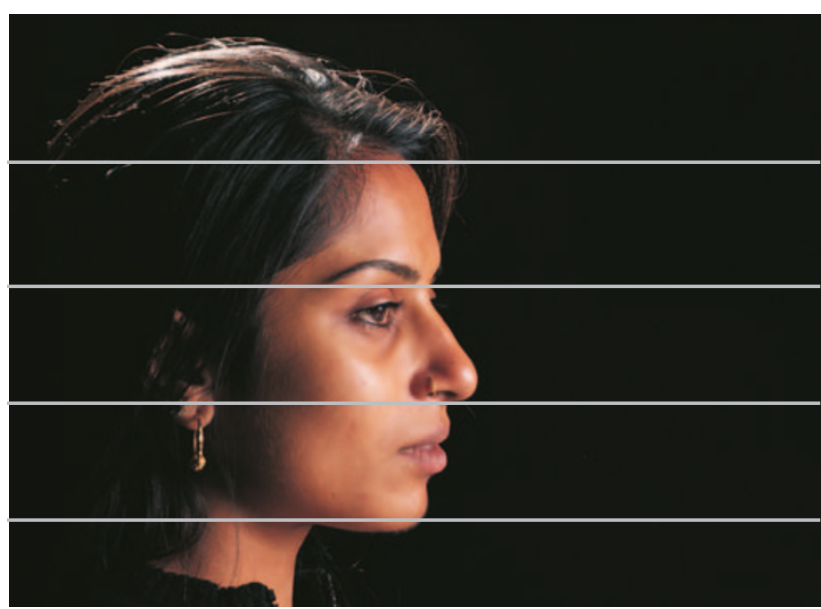

Fig. 11 Sagittal view of the three facial divisions 
Fig. 12

Morphopsychological equilibrium is realised when the facial thirds are equal both quantitatively and qualitatively

Fig. 13 Asymmetrical right and left facial halves (hemiface)
To achieve equilibrium and harmonious integration, the facial zones (Fig. 12) should be equal quantitatively (morphologically) and qualitatively (psychologically). If one of the zones is physically larger, say the middle third (emotional), another can compensate for this qualitatively, without creating imbalance. In the latter example, placing larger teeth in the lower third of the face would counterbalance the physically larger middle third segment.
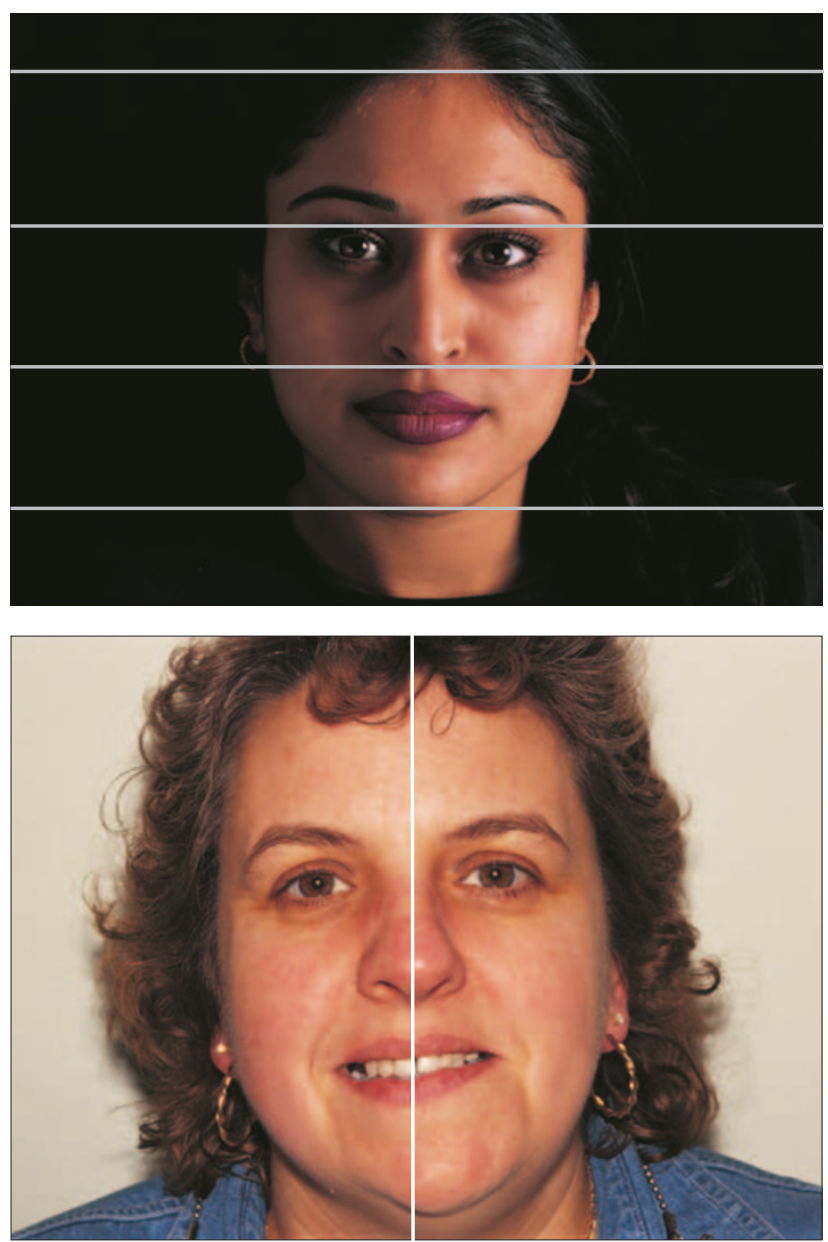

Each of the facial zones is assigned a sense organ (or receptor), the upper third: the eyes, the middle: the nose and the lower: the lips. The vestibular frame of the facial map of the face encloses these receptors, which can be either open or closed. Wide eyes, dilated nostrils and voluptuous lips have obvious psychological significance of welcome, arousal and sensuality, respectively. Conversely, closed receptors convey the opposite connotations of alienation, passivity and frigidity. A wide, curved, round angled tooth form is appropriate for patients with open receptors and vice versa for those with closed receptors.

The degree of tegumental relief is inherited, but laxness increases with advancing years. Rough, pitted and textured skin requires teeth with similar characteristics, while smooth silky soft tissue yearns for a glossy, smooth dentition. In addition, younger patients with increased skin tone should have restorations that correspond to their vitality, while older generations should have prostheses that convey wear and maturity, conforming to reduced tone and more wrinkles.

Qualities assigned to biological masculinity and femininity are blatant. Masculine facial features display prominent osseous structures, angular jaw lines, closed facial angle, and rectangular soft tissue angles, etc. Feminine components encompass delicate osseous make-up, oval jaws, open facial angle and rounded soft tissues angles, etc. However, in contemporary society and aberrant life styles, the conventional sexual types may be ambivalent. Therefore, the clinician should ascertain the biological, as well as preferred sexual type, before prescribing anterior restorations, which may conflict with the patient's overt sexuality.

Finally, hemifaces describe asymmetry of the right and left sides of a face ${ }^{7}$ (Fig. 13). This may be inherited, sequalae of trauma, or determined by disease or psychologically. Inheritance plays a major role for dental element form, alignment, colour, etc; the maxillary right and left anterior sextant is rarely identical (Fig. 14) which does not often cause visual tension, but adds diversity and interest to a dental composition. Accidents and disease may cause severe disfigurement beyond what is aesthetically acceptable, necessitating clinical intervention to resolve the anomalies.

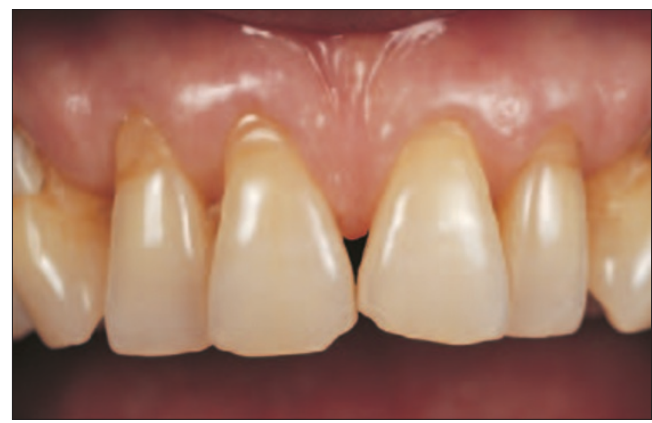

Fig. 14 Asymmetrical right and left anterior maxillary sextant

Lastly, from a psychological aspect, the right and left brain hemispheres are responsible for different cerebral activities. For a right-handed person, the left side of the brain, controlling the right part of the face, is responsible for cognitive, computational and practical activities, while the right side (controlling the left part of the face) for creative and conceptual ideas. A sentimental social disaster may result in sagging (reduced muscle and skin tonicity) to the left facial middle third (ascribed to emotions and feelings), which could be compensated by providing appropriate anterior teeth in the maxillary anterior right sextant.

\section{GEOMETRIC}

The final, and most objective, method of facial assessment is based on mathematical principles of evaluating beauty. Methods for quantifying beauty preoccupied ancient Greeks, and have mystified philosophers and scientists throughout the centuries. A review of the dental literature 
shows numerous, ingenious criteria for dental harmony which complement facial features. ${ }^{8}$ None of these observations has scientific foundation, since facial features and tooth form are determined genetically. ${ }^{9}$ The facial characteristics of a sibling may be inherited from one parent, while tooth morphology from the other parent. Therefore, facial characteristics have no correlation to tooth shape, size, colour, alignment, etc. However, there is consensus that certain proportions (both facial and dental) are psychologically perceived as visually pleasing. This dictum is the basis of mathematical analysis of facial attractiveness.

The following discussion on geometrical evaluation should be regarded as a general framework, since an object created with mathematical precision, which is scarcely observed in nature, will undoubtedly be perceived as artificial. The reason is that such perfection is a rarity, and sensed as a fake or simulation. The latter is particularly true of computer graphics, which try to emulate objects and beings with precision, ignoring nuances prevalent in the natural world.

While ideal facial features may be related to the Golden proportion, they have little significance in dentistry. However, in plastic or reconstructive surgery, facial landmarks according to the Golden proportion are valuable guidelines for the surgical team. A geometric evaluation of the face is visualising imaginary lines in the frontal and sagittal views. Commencing from the upper to the lower parts of the face, the horizontal lines are:

- Hair

- Ophriac

- Interpupillary

- Interalar

- Commissural.

These parallel lines create horizontal symmetry and act as cohesive forces unifying the facial composition. The facial midline is perpendicular to the horizontal lines and opposes their cohesiveness. The latter are termed segregative forces and are essential in a composition to give it interest and harmony (Fig. 15). The cohesive forces are paramount in achieving pleasing aesthetics; the deviation of the facial midline is secondary and varies in many individuals without a deleterious effect. It is the general parallelism of the horizontal lines, which is important, as opposed to the orientation of one single line. ${ }^{10}$ The interpupillary line is used as a reference for the occlusal and incisal plane orientations. The other horizontal lines can be eschewed and therefore do not act as definite references; they are however, useful accessory markers (Fig. 16). The incisal edges of the anterior teeth should be parallel to the interpupillary line and perpendicular to the midline (Fig. 17). If the incisal plane is tilted this could be attributed to dental or skeletal factors. The dental factors that influence the incisal table are attrition, erosion (e.g. gastric regurgitation as in bulimia), abrasion, altered patterns of eruption and periodontal disease. If the latter are eliminated, then

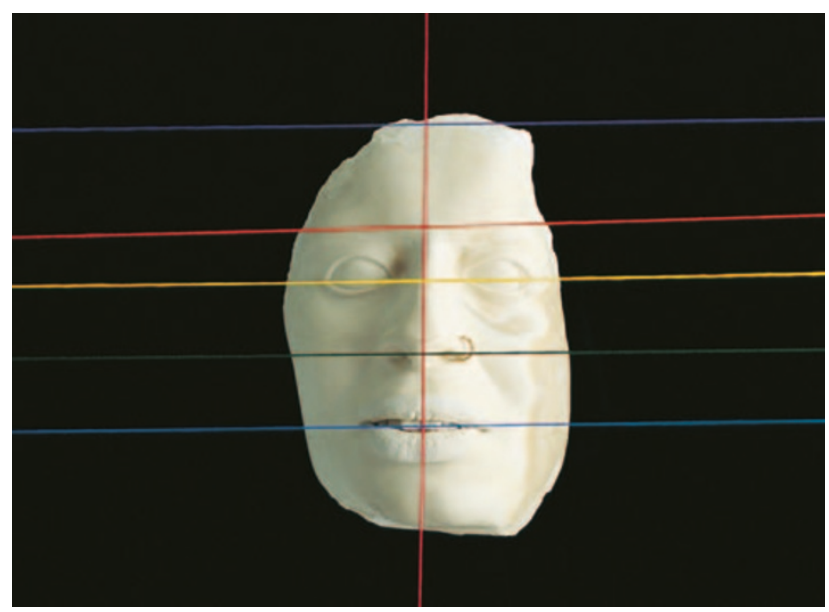

Fig. 15 Horizontal and vertical facial lines in the front view

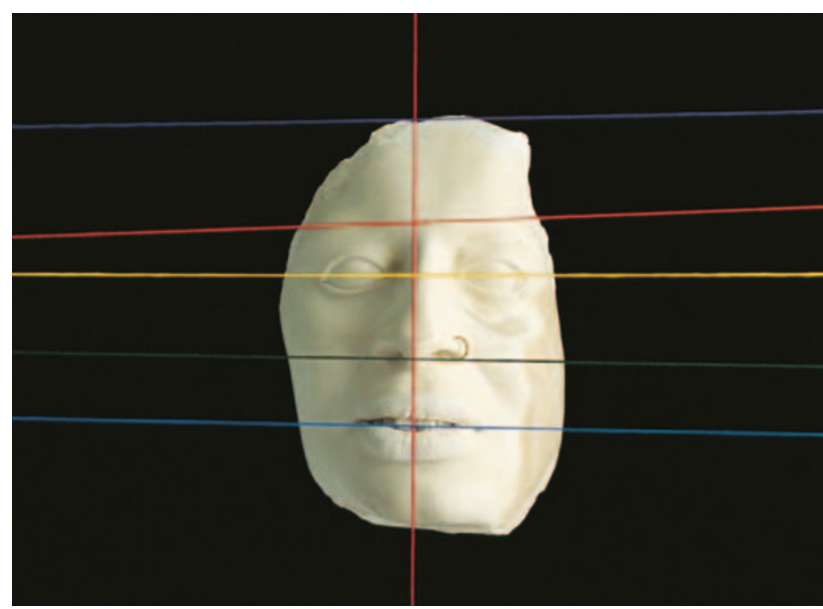

Fig. 16 It is not obligatory for all the facial horizontal lines to be parallel to gain aesthetic approval

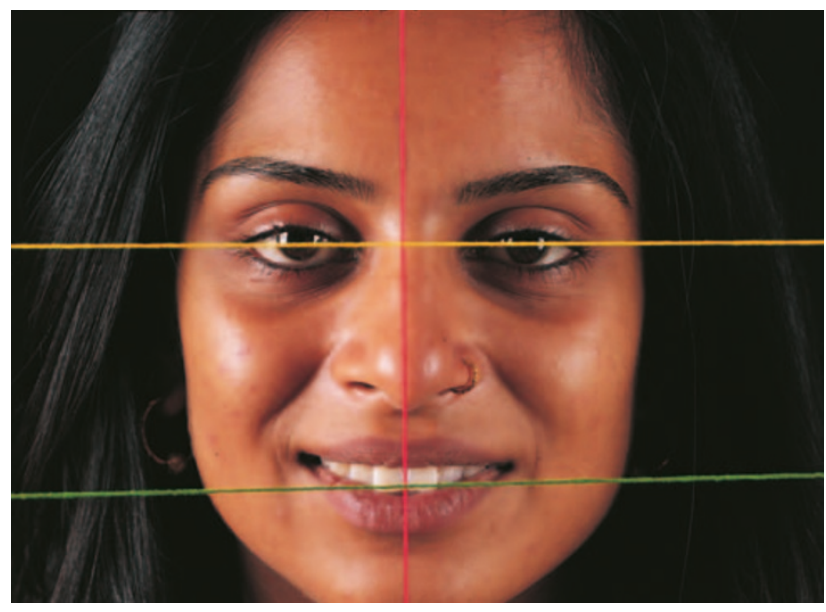

Fig 17. Ideally, the interpupillary line should be parallel to the incisal plane and perpendicular to the facial midline

the tilting could be due to a slanted maxilla. It is vital to determine which factor is responsible for the misalignment of the incisal plane, as this will have a profound impact on the proposed treatment plan. The frontal view also enables assessment of whether the patient has an open or closed bite, following posterior tooth loss, resulting in a diminished linear dimension of the lower third of the face.

As mentioned above, the interpupillary and incisal lines are essential reference markers in prosthodontics. The latter are conveyed to the ceramist for articulation by means of a facebow transfer. Caution is advised when using a facebow, since the right and left soft tissue auditory meatus may be at different levels. A discrepancy in the intercondylar axis results in 
Fig. 18 Correctly positioned facebow viewed from frontal aspect

Fig. 19 Correctly positioned facebow viewed from sagittal aspect

Fig. 20 Applicator stick bite parallel to interpupillary line viewed from frontal aspect

Fig. 21 Applicator stick bite parallel to interpupillary line viewed from sagittal aspect differences between the functional and aesthetic planes of occlusion. The best method to avoid this inaccuracy is by aligning the facebow so that it coincides with the interpupillary line (Figs 18 and 19). Other auxiliary methods, complementing a facebow, are using either a spirit level or an applicator stick bite (Figs 20 and 21). The case study in Figures 22 and 23 shows a clinical example for rectifying disparate interpupillary and incisal plane lines. (The book version of this article will show a detailed clinical sequalae for achieving the latter).
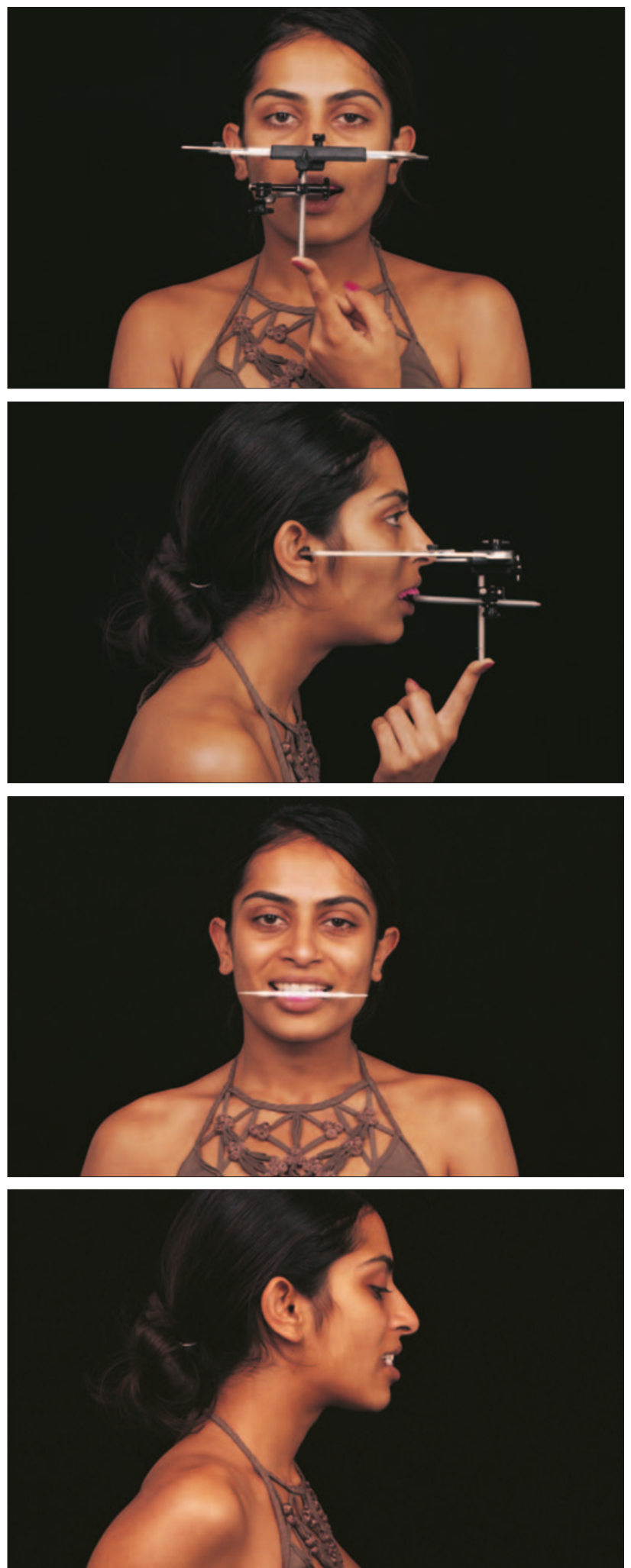

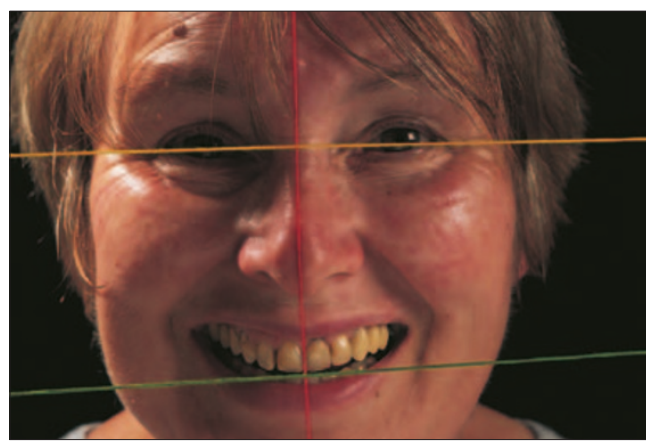

Fig. 22 Pre-operative status showing disharmony between interpupillary and incisal plane lines

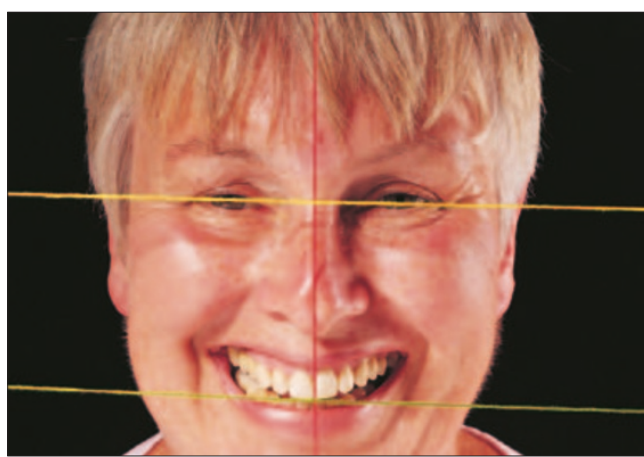

Fig. 23 Post-operative status showing coincidence between interpupillary and incisal plane lines

From the sagittal aspect, the horizontal lines also reinforce the cohesiveness of the profile (Fig. 24). In addition, the Frankfurt horizontal plane, Rickett's E-plane, ${ }^{11}$ the Steiner ${ }^{12}$ or Burstone ${ }^{13}$ lines and the nasolabial angle also contribute to profile assessment. Two are considered below in detail, the Rickett's E-plane and the nasolabial angle, which ascertain the protrusion or retrusion of the maxilla as well as lip competence. In addition to soft tissue analysis, cephalometric reference points are invaluable for assessing the profile. The latter will distinguish between soft tissue and skeletal abnormalities and reveal Angle, Class I, II or III jaw relationships. ${ }^{14}$

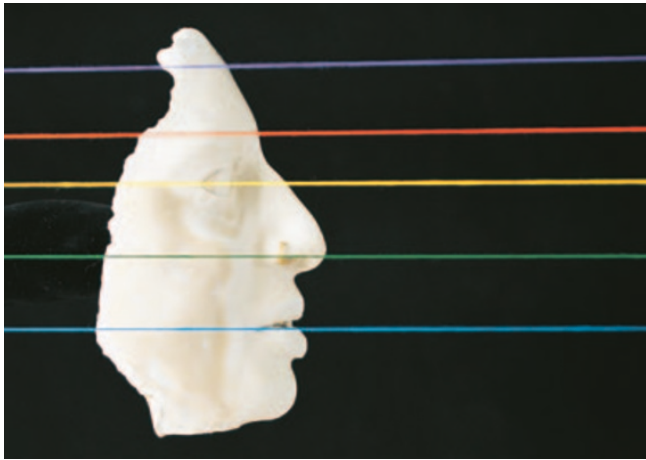

Fig. 24 Horizontal facial lines viewed from sagittal

The nasolabial angle is the intersection of two lines using the nose and lips as reference points. The two lines are tangents of the subnasale and the maxillary lip profile (Fig. 25). For males, this angle rages from $90^{\circ}$ to $95^{\circ}$ and for females from $100^{\circ}$ to $105^{\circ}$. Assuming $90^{\circ}$ as the normal, if the nasolabial angle is 
$<90^{\circ}$, the maxilla is prominent and a convex facial profile is manifested. In this case, recessed maxillary anterior restorations are considered. If the reverse is present, manifesting a concave profile, i.e. nasolabial angle is $>90^{\circ}$, a prominent maxillary anterior dental segment is indicated. Spear ${ }^{15}$ has termed this concept of facial profile to determine the position, and degree of dominance, of the maxillary anterior teeth as 'facially generated treatment planning'.

The Rickett's E-plane is a line drawn from the nose tip to the chin prominence. Accepted norms for the distance from the maxillary lip to this imaginary line are $4 \mathrm{~mm}$, while that for mandibular lip is $2 \mathrm{~mm}$ (Fig. 26). With edentulous cases, it is imperative to restore the Rickett's E-plane to establish a correct facial profile. For example, in edentulous cases, the maxillary lip is unsupported and the measurement to the E-plane is greater than $4 \mathrm{~mm}$, indicative of a deficient facial profile (Fig. 27). Consequently, when artificial prostheses are fabricated, the latter should re-establish lip support and restore the facial profile (Fig. 28).

\section{CONCLUSION}

Facial assessment is both subjective and objective. Physiognomy and morphopsychology are subjective methods of appraisal, while geometric is an objective analysis. It is the combination of the two that achieves dental restorations which integrate with facial features and are perceived with aesthetic approval. However, the ultimate result is heavily dependant on the clinician's and the ceramist's creativity and artistic input.

1. Jung CG. Dreams. Princeton University Press, Princeton, New Jersey, 1974.

2. Myers DG. Psychology, Worth Publishers, Inc., New York, 1998

3. Hassin $R$, Trope $Y$. Facing faces: studies on the cognitive aspects of physiognomy. J Pers Soc Psycho/ 2000; 78 : 837-852.

4. Armstrong E. The limbic system and culture: an allometric analysis of the neocortex and limbic nuclei. Human Nature 1990.

5. McGarth C, Lui KS, Lam C W. Physiognomy and teeth: An ethnographic study among young and middle-aged Hong Kong adults. Br Dent J 2002; 192 (9): 522-525.

6. Rufenacht CR. Fundamental of Esthetics. Quintessence Publishing Co. Inc., Chicago, II, 1990.

7. Gebhard W. A comprehensive approach for restoring esthetic and function in fixed prosthodontics. ODT 2003: 26: 21-44.

8. Levin E I. Dental aesthetics and the golden proportion. J Prosthet Dent 1978; 40: 244-252.

9. Lehman W. Tooth form and the face: A comedy of errors. South CalifState Dent J 1950: 17:29.

10. Chiche, GJ and Pinault A. Esthetics of Anterior Fixed Prosthodontic. Quintessence Pub Co.Inc., 1994: 1; 15.

11. Levin JB. Esthetic diagnosis. Current Opinion in Cosmetic Dentistry, Current Science, 1995: 9-17.

12. Weickersheimer P B. Steiner analysis. In: Jacobson A ed. Radiographic Cephalometry. Carol Stream, II: Quintessence Publishing: 1995: 83-85.

13. Burstone $\mathrm{C}$ J. Lip posture and its significance in treatment planning. Am J Orthod 1967; 53: 262-284.

14. Rifkin R. Facial analysis: A comprehensive approach to treatment planning in aesthetic dentistry. Pract Periodont Aesthet Dent 2000; 12 (9): 865-871.

15. Spear F. Creating Esthetic Excellence Part I, A Complete Approach. Presented at the ADA Meeting in Las Vegas, 1995.

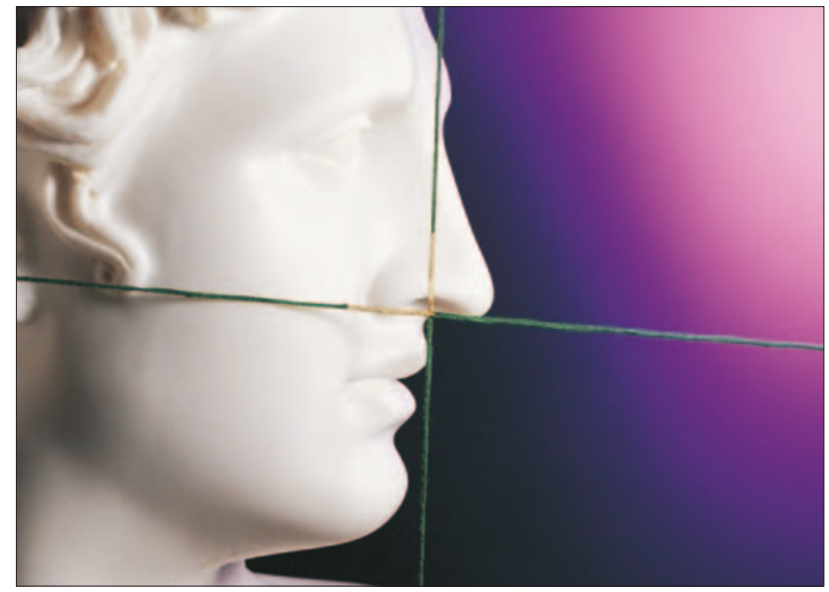

Fig. 25 Nasolabia angle (gold coloured)

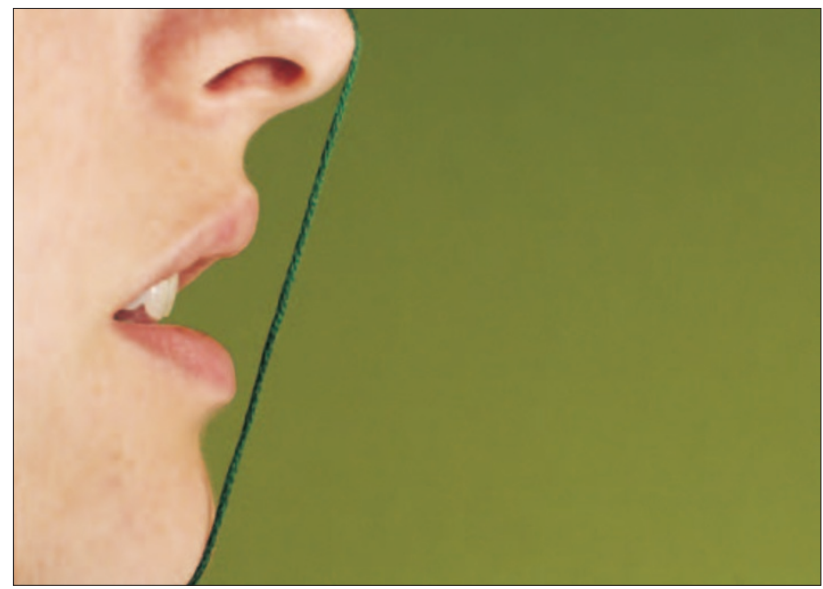

Fig. 26 Rickett's

E-plane

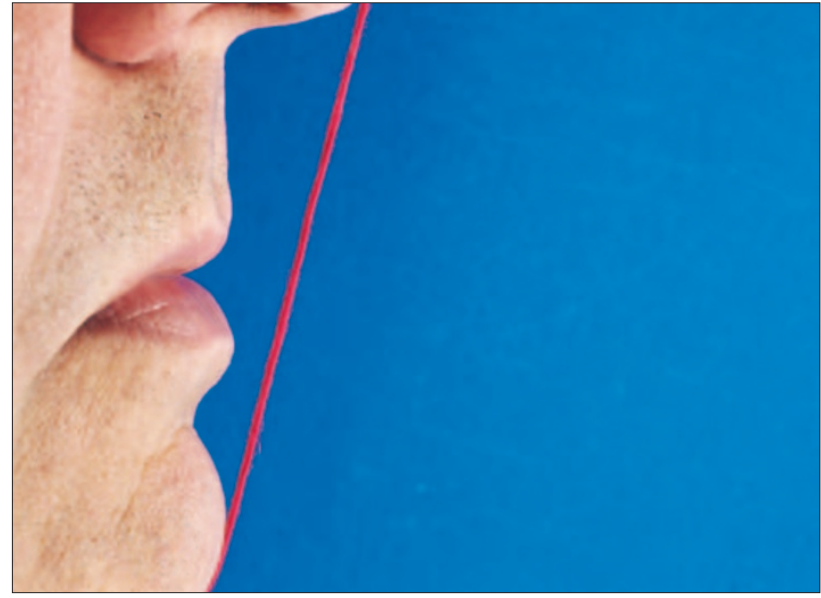

Fig. 27 Unsupported maxillary lip resulting in a deficient facia profile

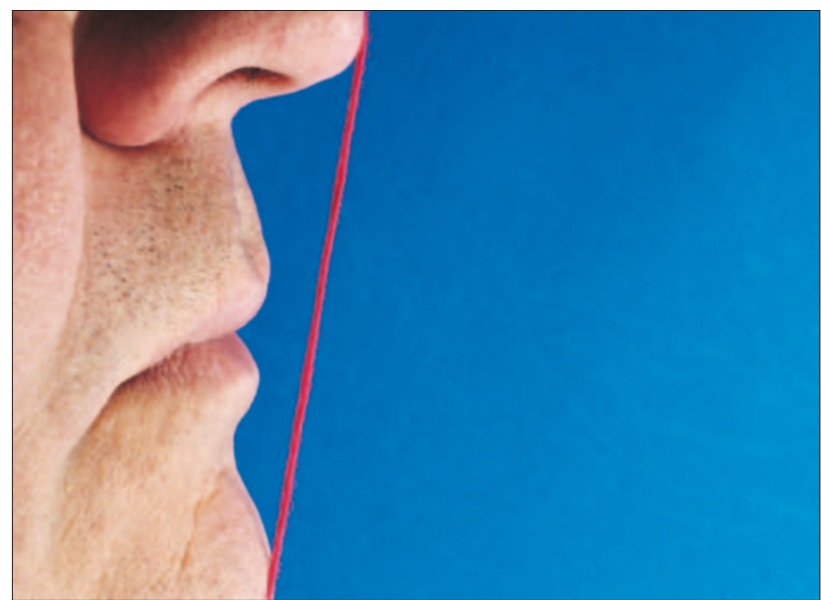

Fig. 28 Following provision of maxillary prostheses, the upper lip is now supported restoring the facial profile (compare with Fig. 27) 